\title{
Anti-A2/RA33 Autoantibodies Are Directed to the RNA Binding Region of the A2 Protein of the Heterogeneous Nuclear Ribonucleoprotein Complex \\ Differential Epitope Recognition in Rheumatoid Arthritis, Systemic Lupus Erythematosus, and Mixed Connective Tissue Disease
}

\author{
Karl Skriner, ${ }^{\star \ddagger}$ Wolfgang H. Sommergruber, ${ }^{\S}$ Viktor Tremmel, ${ }^{\star \ddagger}$ Inge Fischer, ${ }^{\ddagger}$ Andrea Barta, ${ }^{\star}$ Joseph S. Smolen, \\ and Günter Steiner*\#\| \\ *Institute of Biochemistry, A-1030 Vienna; ${ }^{\ddagger}$ Ludwig Boltzmann-Institute for Rheumatology and Balneology, A-1106 Vienna; ${ }^{\S}$ Bender

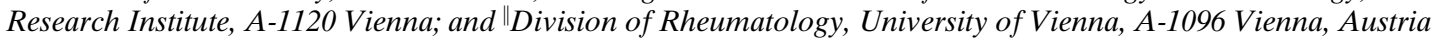

\begin{abstract}
The recently described anti-A2/RA33 autoantibodies occur in $20-40 \%$ of patients with RA, SLE, and mixed connective tissue disease (MCTD). They are directed to the A2 protein of the heterogeneous nuclear ribonucleoprotein complex (hnRNP-A2), an abundant nuclear protein associated with the spliceosome. The $\mathrm{NH}_{2}$-terminal half of the antigen contains two conserved RNA binding domains whereas its $\mathrm{COOH}$-terminal part is extremely glycine-rich. The aim of this study was to characterize the autoepitopes of hnRNPA2 and to investigate the effects of anti-A2/RA33 autoantibodies on possible functions of the antigen. Using bacterially expressed fragments, two major discontinuous epitopes were identified. One containing the complete second RNA binding domain was recognized by the majority of patients with RA and SLE but not by patients with MCTD. The second epitope contained sequences of both RNA binding domains and was preferentially targeted by patients with MCTD. When the RNA binding properties of the antigen were investigated, oligoribonucleotides containing the sequence motif r(UUAG) were found to bind to a site closely adjacent or overlapping with the epitope targeted by autoantibodies from patients with RA and SLE. Moreover, anti-A2/RA33 autoantibodies from patients with RA or SLE, but not from patients with MCTD, inhibited binding of RNA. Thus, anti-A2/RA33 autoantibodies recognize conformation-dependent epitopes located in a functionally important region of the antigen. Furthermore, the specific recognition of an epitope by MCTD patients may be used as another argument in favor of considering MCTD a distinct connective tissue disease. (J. Clin. Invest. 1997. 100:127135.) Key words: anti-A2/RA33 autoantibody - epitope recognition • rheumatoid arthritis $\bullet \mathrm{SLE} \cdot$ mixed connective tissue disease
\end{abstract}

Portions of this work have been presented at the Annual Meetings of the American College of Rheumatology, Minneapolis, October 1994, and Orlando, October 1996.

Address correspondence to Dr. Günter Steiner, Division of Rheumatology, Department of Internal Medicine III, University of Vienna, Währinger Gürtel 18, A-1096 Vienna, Austria. Phone: 43140400-2121; FAX: 431-40400-4306; E-mail: gs@bch.univie.ac.at

Received for publication 2 December 1996 and accepted in revised form 3 April 1997.

J. Clin. Invest.

(C) The American Society for Clinical Investigation, Inc.

0021-9738/97/07/0127/09 \$2.00

Volume 100, Number 1, July 1997, 127-135

\section{Introduction}

A characteristic feature of rheumatic autoimmune diseases such as SLE, progressive systemic sclerosis, polymyositis, mixed connective tissue disease (MCTD) ${ }^{1}$, or RA is the occurrence of autoantibodies to intracellular antigens $(1,2)$. For reasons which are not yet fully understood these autoantibodies are often directed to components of large ribonucleoprotein (RNP) structures such as the ribosome or the spliceosome (3). Some of these autoantibodies specifically occur in only one disease which makes them very useful for diagnosis. Thus, autoantibodies to double-stranded DNA or to the Sm antigen are highly specific for SLE, autoantibodies to topoisomerase (anti-Scl70) are exclusively detected in patients with progressive systemic sclerosis, and autoantibodies to tRNA synthetases (e.g., anti-Jo1) occur only in patients with poly- or dermatomyositis.

In contrast to these disorders, pathognomonic autoantibodies have not yet been defined in RA. So far, rheumatoid factor, an autoantibody directed to the Fc region of human $\mathrm{IgG}$, is still the only well established serological disease marker (4). However, rheumatoid factor is not specific for RA and is often negative in the early stages of the disease when a definite diagnosis is not always possible. In the past few years several new autoantibodies have been described which may be more specific for RA than rheumatoid factor. Among these are anti-A2/ RA33 antibodies $(5,6)$, antikeratin antibodies and the antiperinuclear factor $(7,8)$, and anti-Sa antibodies (9).

Anti-A2/RA33 autoantibodies have been shown to target the A2 protein of the heterogeneous nuclear RNP (hnRNP) complex (10). This complex is composed of pre-mRNA and $\sim 30$ different proteins and functionally forms part of the spliceosome (11). Interestingly, spliceosome-associated antigens, such as the U1 small nuclear ribonucleoprotein (U1-snRNP) or the Sm proteins, are typically targeted by patients with SLE and MCTD, but not by patients with RA $(1,2)$. Thus, the hnRNP A2 protein (hnRNP-A2) (together with its alternatively spliced variants hnRNP-B1 and -B2, and the closely related hnRNP-A1) is so far the only known spliceosome-associated autoantigen which is recognized by patients with RA, emphasizing the immunological relationship between RA, which primarily affects the joints, and the more systemic diseases SLE and MCTD (12, 13). Anti-A2/RA33 autoantibodies are not strictly specific for RA as they are found not only in

1. Abbreviations used in this paper: hnRNP, heterogeneous nuclear ribonucleoprotein; hnRNP-A2, A2 protein of the heterogeneous nuclear ribonucleoprotein; MCTD, mixed connective tissue disease; RBD, RNA binding domain; RNP, ribonucleoprotein; snRNP, small nuclear ribonucleoprotein. 
$\sim 35 \%$ of patients with RA, but also in $\sim 20 \%$ of patients with SLE and in 40-60\% of patients with MCTD $(13,14)$. However, in SLE (and MCTD) they usually occur together with antibodies to U1-snRNP or Sm. Therefore, anti-A2/RA33 without concomitant anti-U1-snRNP autoantibodies were found to have $96 \%$ specificity for RA (13).

hnRNP-A2 is one of the core proteins of the hnRNP complex (15). Apart from its general functions in binding and packaging of pre-mRNA, hnRNP-A2 seems to be involved in mRNA transport and the regulation of alternative splicing (16, 17). Interaction with RNA is mediated by two adjacent conserved RNA binding domains (RBD), each 86 amino acids long, located in the $\mathrm{NH}_{2}$-terminal half of the protein (18). RBDs of similar structure are contained in many RNA binding proteins, among those important SLE autoantigens such as the U1 RNA associated proteins $\mathrm{U} 1-70 \mathrm{~K}$ and U1-A, or the hY RNA associated antigens Ro/SS-A and La/SS-B. The COOHterminal part of hnRNP-A2, the auxiliary domain, contains $\sim 50 \%$ glycine residues and is presumably involved in interactions with other proteins.

In this study, we have analyzed epitope recognition by antiA2/RA33 autoantibodies. We demonstrate that the major antibody binding sites are conformation dependent and located in the $\mathrm{NH}_{2}$-terminal RNA binding region of hnRNP-A2 and that patients with RA and SLE differ in epitope recognition from patients with MCTD. Furthermore we show that the epitope recognized preferentially by patients with RA and SLE is also indispensable for binding of RNA and that antiA2/RA33 autoantibodies are able to inhibit binding of RNA in vitro.

\section{Methods}

Patients. For epitope mapping studies, 32 sera showing pronounced anti-A2/RA33 autoreactivities by immunoblotting were selected from anti-A2/RA33 positive patients with RA $(n=14)$, SLE $(n=$ $10)$, and MCTD $(n=8)$. For each disease category, five anti-A2/ RA33 negative patients were used as controls and five sera from healthy individuals served as additional controls. Patients were selected from a cohort of patients clinically and serologically well characterized previously (13). Selection was made on the following grounds. RA sera were randomly selected; SLE sera were selected to include $50 \%$ of patients without autoreactivity to U1-snRNP and/or Sm taking into account the strong association of these reactivities with anti-A2/RA33 antibodies (13); and the eight anti-A2/RA33 positive MCTD sera selected were those ones available in sufficient amounts. All RA patients fulfilled the 1987 revised criteria of the American College of Rheumatology (19), all SLE patients the 1982 criteria of the American College of Rheumatology (20), and all MCTD patients the criteria of Alarcón-Segovia and Villareal (21). Although MCTD patients fulfilled between two and four of the SLE criteria none of them suffered from renal disease or showed anti-dsDNA antibodies (13). All MCTD patients had long-standing disease and have not progressed to SLE or RA in the past $6 \mathrm{yr}$.

In addition to anti-A2/RA33, the 10 SLE patients selected had autoantibodies to $\operatorname{Sm}(n=3)$, U1-snRNP $(n=3)$, Ro/SSA $(n=5)$, $\mathrm{La} / \mathrm{SSB}(n=2)$, and ribosomal RNP $(n=1)$. Eight patients had antidsDNA antibodies. All MCTD patients had high-titer antibodies to U1-snRNP and recognized the three U1-snRNP associated antigens $70 \mathrm{~K}$, A, and $\mathrm{C}$ by immunoblotting; this serologic feature characteristic of MCTD was also seen in one patient with SLE. However, this patient fulfilled eight criteria for SLE of the ACR including nephritis, hypocomplementemia, and anti-DNA antibodies and therefore could not be classified as MCTD either on clinical or on serological grounds.
In patients with RA no antibody other than anti-A2/RA33 was detected, except for rheumatoid factor which was present in 10 of the 14 sera. Rheumatoid factor was also present in six SLE and five MCTD patients.

Oligonucleotides. Oligoribonucleotides and PCR primers were obtained from the DNA synthesis and sequencing unit of the Vienna Biocenter.

Cloning and expression of hnRNP-A2 deletion mutants. The cDNA encoding the entire sequence of hnRNP-A2 (kind gift of C. Burd, Howard Hughes Medical Institute, Philadelphia, PA) was cloned into the expression vector pEx 34 (22) and expressed upon temperature induction as fusion protein with MS-2 polymerase. A series of deletion mutants were constructed by limited Bal-31 digestion of the cDNA from the BamHI site located in the coding region (amino acid 41) and from the NsiI site located in the $3^{\prime}$ untranslated region. Clones of interest were sequenced and transfected into the Escherichia coli 537 strain. Production of fusion proteins was induced by incubating the cells for $2 \mathrm{~h}$ at $42^{\circ} \mathrm{C}$. For expression of A2 as a nonfusion protein, a DNA fragment encoding the $\mathrm{NH}_{2}$-terminal region (amino acids 1-41) was generated by PCR (using the oligonucleotides GTCCGCCATGGAGAGAGAAAAGGA and TGCAGGATCCCTCATTACCACACAGTCTGT as primers) and ligated to the fragment comprising the rest of the cDNA coding region (amino acids 42-341). Subsequently, the DNA was cloned into the pET 3d vector (New England Biolabs Inc., Beverly, MA) and expressed in the E. coli BL21 (De3) pLysS strain by induction for $3 \mathrm{~h}$ with $0.4 \mathrm{mM}$ IPTG.

Purification of hnRNP-A2 deletion mutants. Recombinant proteins were prepared by resuspending the cell pellet obtained from 0.5 liter cultures in buffer A ( $25 \mathrm{mM}$ Na-acetate, $2 \mathrm{M}$ urea, $0.01 \%$ Triton $\mathrm{X}-100, \mathrm{pH} 5.5$ ) followed by sonication and centrifugation at 18,000 $\mathrm{rpm}$ for $20 \mathrm{~min}$ in a Sorvall SS34 rotor. The pellets containing the inclusion bodies were resuspended in $8 \mathrm{ml}$ of buffer B $(25 \mathrm{mM}$ Na-acetate, $8 \mathrm{M}$ urea, $\mathrm{pH} 5.5$ ), sonicated again, and applied to a 5-ml carboxymethyl-Sepharose column (Pharmacia Biotech AB, Uppsala, Sweden) equilibrated with buffer B. After washing the column with 5 vol of buffer B, proteins were eluted stepwise with buffer B containing either $50,75,100,150$, or $200 \mathrm{mM} \mathrm{NaCl}$. Recombinant proteins were recovered from the $150 \mathrm{mM} \mathrm{NaCl}$ fraction and dialyzed against buffer $\mathrm{C}(20 \mathrm{mM}$ Hepes-NaOH, pH $8.0,100 \mathrm{mM} \mathrm{KCl}, 0.2 \mathrm{mM}$ EDTA, $5 \%$ (vol/vol) glycerol, $1 \mathrm{mM} \mathrm{DTT}$ ). Purity was $\sim 95 \%$ as analyzed by SDS-PAGE and Coomassie protein staining. Protein concentration was measured in a microplate reader at $620 \mathrm{~nm}$ using the Coomassie protein assay reagent from Pierce Chemical Co. (Rockford, IL).

Proteolytic digest of recombinant hnRNP-A2. Since the $\mathrm{COOH}-$ terminal part of hnRNP-A2 could be only poorly expressed, purified recombinant hnRNP-A2 was digested with V8 protease taking advantage of the fact that hnRNP-A2 contains several V8 specific cleavage sites in the $\mathrm{NH}_{2}$-terminal region but none in the $\mathrm{COOH}$-terminal part. Thus, by incubating $100 \mu \mathrm{g}$ protein with $10 \mu \mathrm{g}$ V8 protease (Boehringer Mannheim, Mannheim, Germany) for $24 \mathrm{~h}$ at room temperature in digestion buffer $(50 \mathrm{mM}$ ammonium bicarbonate, $0.025 \%$ SDS, $1 \mathrm{mM} \mathrm{CaCl}$, pH 7.8) the $\mathrm{NH}_{2}$-terminal part was degraded whereas the $\mathrm{COOH}$-terminal part of the protein (amino acids 185341) remained intact. This fragment was purified by SDS-PAGE and its reactivity with autoantibodies was tested by immunoblotting.

Gel electrophoresis and immunoblotting. Samples were separated on $12 \%$ SDS minigels $(8 \times 5 \mathrm{~cm} \times 0.75 \mathrm{~mm})$. Electrophoretic transfer to nitrocellulose (BAS53; Schleicher und Schüll, Dassel, Germany) was performed for $60 \mathrm{~min}$ at $400 \mathrm{~mA}$. After blocking the nitrocellulose with blocking buffer (3\% nonfat dried milk in PBS, pH 7.4) for 60 min, the blots were incubated for 30 min with sera diluted between 1:25 and 1:100 in the same buffer. Although higher serum dilutions could be used when the buffer contained detergents such as $0.1 \%$ Triton X-100 or Tween 20 these substances were avoided during the blocking and incubation steps since they can cause false positive results by favoring nonspecific binding of DNA-anti-DNA immune complexes frequently present in sera of SLE patients (23). The blots were 
washed $3 \times 5$ min with PBS containing $0.1 \%$ Triton X-100 (PBS-Triton) and subsequently incubated for $30 \mathrm{~min}$ with an alkaline phosphatase-coupled anti-human IgG (Fc) second antibody (Accurate Chemical and Science Co., Westbury, NY) diluted 1:2,500 in PBS-Triton, $3 \%$ nonfat dried milk. The recombinant full-size protein was always run in parallel with the deletion mutants as a positive control and reactivity of the mutants was estimated semiquantitatively $(+++,++,+,-)$ by three independent observers (K. Skriner, I. Fisher, and G. Steiner).

Immunoaffinity purification of antibodies. For RNA competition experiments (see below), anti-A2/RA33 autoantibodies from two RA and one SLE patient (who was also anti-Sm positive) were affinitypurified by the blot elution technique as described (13). Anti-Sm $\left(\mathrm{B} / \mathrm{B}^{\prime}, \mathrm{D}\right)$ autoantibodies purified by the same method were used as controls. Briefly, nitrocellulose strips containing the blotted antigens were incubated overnight at $4^{\circ} \mathrm{C}$ with sera diluted $1: 10$ in blocking buffer, washed thoroughly, cut into small pieces, and finally incubated for 2 min with $0.1 \mathrm{M}$ Tris, $\mathrm{pH} 10.5$. The eluate was immediately neutralized with $1 / 10$ vol of $1 \mathrm{M}$ Tris- $\mathrm{HCl}, \mathrm{pH}$ 6.8. Eluted antibodies were concentrated in Centricon 30 tubes (Amicon Inc., Beverly, MA) by centrifugation for $20 \mathrm{~min}$ at $5,000 \mathrm{~g}$. To estimate the yield, affinitypurified antibodies were separated on SDS gels and stained with Coomassie blue using purified human IgG (Sigma Chemical Co., St. Louis, MO) as standard. Finally, eluted antibodies were diluted to a concentration of $\sim 100 \mathrm{ng} / \mathrm{ml}$ in blocking buffer and analyzed by immunoblotting using either nuclear extracts or purified hnRNP-A2 as antigen.

Northwestern analysis. Approximately $0.5 \mu \mathrm{g}$ of the purified recombinant proteins was separated on a $12.5 \%$ SDS polyacrylamide gel and transferred onto nitrocellulose by electroblotting. The proteins were allowed to renature for $18-24 \mathrm{~h}$ with three changes of renaturation buffer $(10 \mathrm{mM}$ Tris- $\mathrm{HCl}, \mathrm{pH} 7.5,150 \mathrm{mM} \mathrm{NaCl}, 10 \mathrm{mM}$ $\mathrm{MgCl}_{2}, 10 \mathrm{mM} \mathrm{ZnCl}, 10 \%$ glycerol, 5\% BSA, 2.5\% NP-40, $1 \mathrm{mM}$ DTT). After renaturation, the blots were probed with $100 \mathrm{ng}$ of ${ }^{32} \mathrm{P}$ end-labeled $\mathrm{r}(\mathrm{UUAGGG})_{4}$ oligonucleotide in $10 \mathrm{ml}$ assay buffer (10 mM Tris- $\mathrm{HCl}, \mathrm{pH} 7.5,50 \mathrm{mM} \mathrm{NaCl}, 10 \mathrm{mM} \mathrm{MgCl}, 1 \mathrm{mM}$ DTT, $0.25 \% \mathrm{BSA}$ ) for $3 \mathrm{~h}$. Blots were washed three times for $30 \mathrm{~min}$ with washing buffer $(10 \mathrm{mM}$ Tris- $\mathrm{HCl}, \mathrm{pH} 7.5,150 \mathrm{mM} \mathrm{NaCl}, 10 \mathrm{mM}$ $\mathrm{MgCl}_{2}, 1 \mathrm{mM}$ DTT), and exposed to x-ray film. For quantitative analysis an electronic autoradiography system (InstantImager; Packard, Meriden, CT) was used.

$R N A$-protein cross-link assay. Equimolar amounts $(0.3 \mu \mathrm{M})$ of ${ }^{32} \mathrm{P}$-labeled RNA oligonucleotide $\mathrm{r}(\mathrm{UUAGGG})_{4}$ and purified recombinant hnRNP-A2 or deletion mutants, respectively, were mixed and incubated at room temperature for $20 \mathrm{~min}$ in $20 \mu \mathrm{l}$ of binding buffer (10 mM Tris- $\mathrm{HCl}, \mathrm{pH} 7.5,1 \mathrm{mM}$ EDTA, $4 \%$ glycerol, $0.1 \%$ Triton $\mathrm{X}-100,10 \mathrm{mM}$ 2-mercaptoethanol). The reaction mixtures were then transferred to a microtiter plate, put on ice, and irradiated with a UV lamp (Stratalinker, Stratagene Inc., La Jolla, CA) at $254 \mathrm{~nm}$ at a dose of $9.9 \mathrm{~mJ} / \mathrm{mm}^{2}$. After addition of Laemmli sample buffer $(50 \mathrm{mM}$ Tris- $\mathrm{HCl}, \mathrm{pH} 6.8,2 \% \mathrm{wt} / \mathrm{vol}$ SDS, $10 \%$ vol/vol glycerol, $5 \% \mathrm{wt} / \mathrm{vol}$ 2-mercaptoethanol, $0.01 \% \mathrm{wt} / \mathrm{vol}$ bromophenol blue, final concentrations) the samples were boiled for $10 \mathrm{~min}$ and analyzed on $10 \%$ SDSpolyacrylamide gels. The gels were then dried and autoradiographed. Competition experiments were performed by preincubating the recombinant protein for $10 \mathrm{~min}$ at room temperature with a 50 -fold excess of unlabeled oligonucleotides before adding ${ }^{32} \mathrm{P}$-labeled r(UUAGGG) $)_{4}$. For antibody competition experiments, various amounts of affinity-purified autoantibodies $(30,60,90,120$, and 150 ng) were mixed with a constant amount $(0.3 \mu \mathrm{M})$ of the labeled oligonucleotide. Subsequently, these mixtures were incubated with $0.3 \mu \mathrm{M}$ purified hnRNP-A2 as described above.

Figure 1. Coomassie blue staining $(A)$ and immunoblot analysis $(B)$ of purified recombinant hnRNP-A2 and deletion mutants. The recombinant proteins were expressed as MS-2 polymerase fusion proteins, for control purposes full-length hnRNP-A2 was expressed both
A

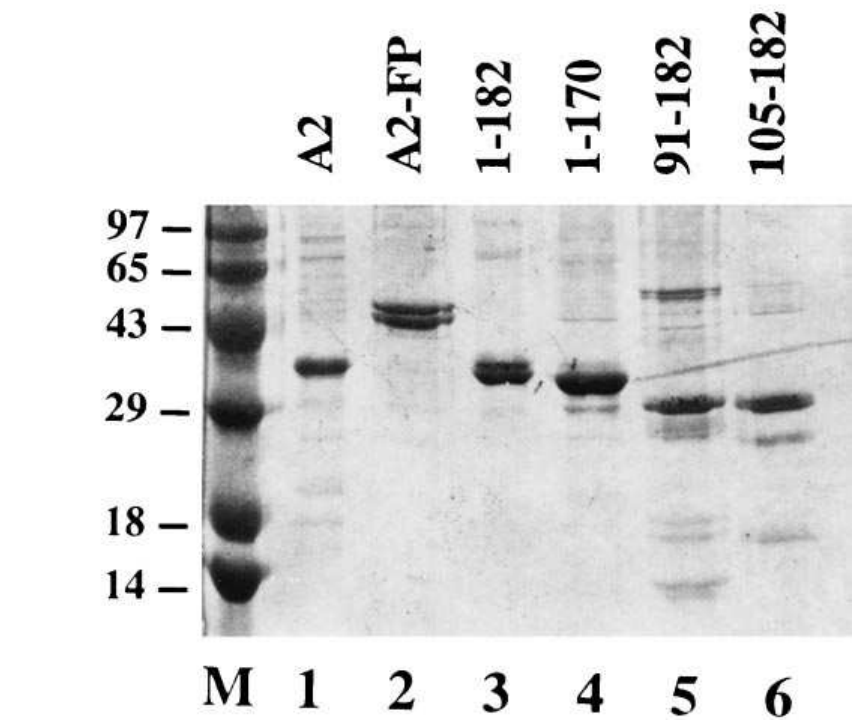

B

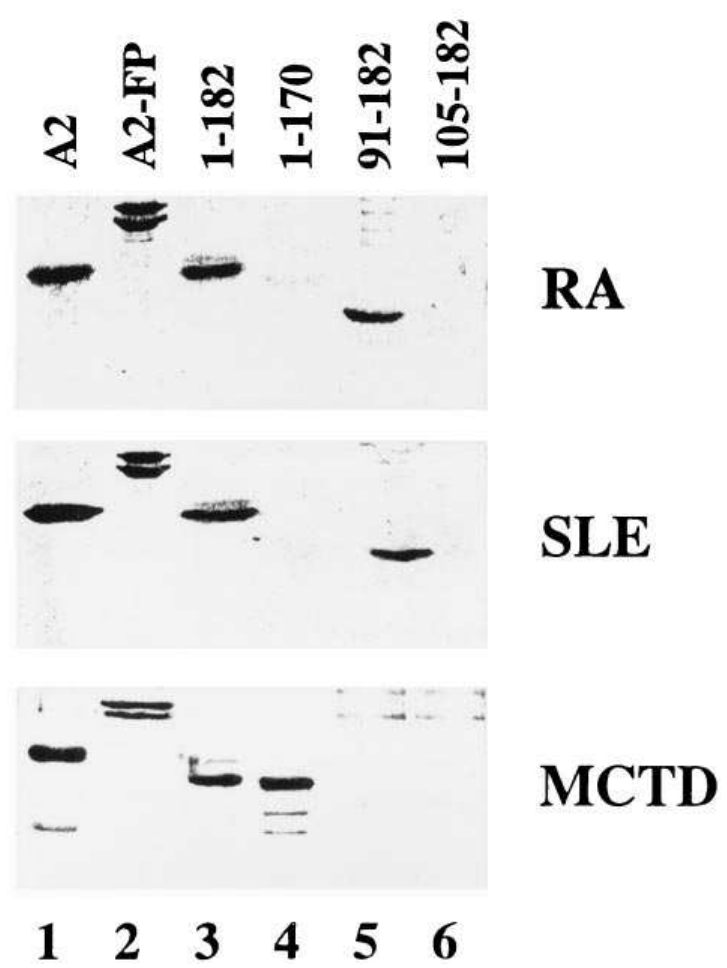

as a nonfusion protein $(A 2$, lane 1$)$ and as fusion protein $(A 2-F P$, lane 2). The positions of the $\mathrm{NH}_{2}$ - and $\mathrm{COOH}$-terminal amino acids of the A2 fragments are indicated on top of the lanes. In $B$, representative immunoblots obtained with three patients' sera (RA, SLE, MCTD) are displayed. Note that the A2-FP (lane 2) usually migrated as double band in SDS gels; this was presumably due to incomplete denaturation of this poorly soluble protein causing aberrantly migrating conformation isomers $(10,13)$. 
Gel retardation assay. To control for artifacts which might be created by UV irradiation, samples were also analyzed on gels run under nondenaturing conditions. The binding and competition assays were performed essentially as described above except that UV irradiation was omitted. Instead, samples were immediately transferred to $5 \%$ nondenaturing polyacrylamide gels run in electrophoresis buffer (25 mM Tris, $192 \mathrm{mM}$ glycine, $\mathrm{pH}$ 6.8) and separated for $60 \mathrm{~min}$ at 10 $\mathrm{V} / \mathrm{cm}$. Subsequently, gels were dried and analyzed by autoradiography.

\section{Results}

Reactivity of patient sera with deletion mutants of hnRNP-A2. To identify the epitopes recognized by anti-A2/RA33 autoantibodies, deletion mutants of recombinant hnRNP-A2 were expressed as fusion proteins with MS-2 polymerase, purified by cation exchange chromatography and separated by SDSPAGE. For control purposes, full-length hnRNP-A2 was expressed both as a nonfusion protein and as a fusion protein. In Fig. $1 A$ Coomassie blue stainings of the full-size nonfusion protein $(A 2$, lane 1$)$, the full-size fusion protein $(A 2-F P$, lane $2)$, and the four most important deletion mutants are shown: mutant 1-182 (lane 3) containing both RBDs (plus five adjacent amino acids); mutant 1-170 (lane 4) lacking the seven $\mathrm{COOH}$-terminal amino acids of the second RBD (RBD II); mutant 91-182 (lane 5) corresponding to RBD II (plus one $\mathrm{NH}_{2}$-terminal and five $\mathrm{COOH}$-terminal amino acids); and mutant 105-182 (lane 6) lacking the $13 \mathrm{NH}_{2}$-terminal amino acids of RBD II. Antigenicity of the protein fragments was tested by immunoblotting using 32 sera from patients with RA, SLE, and MCTD previously characterized as anti-A2/RA33 positive (13). A characteristic result can be seen in Fig. $1 B$ where the reactivity patterns of three individual patients (one RA, one SLE, one MCTD) obtained with the above-mentioned proteins are shown: all three sera were strongly reactive $(+++)$ both with the full-length nonfusion and the fusion protein (lanes 1 and 2) as well as with fragment 1-182 (lane 3). The RA and SLE autoantibodies bound also to fragment 91-182 (lane 5 ), but they recognized neither fragment 1-170 (lane 4) nor fragment 105-182 (lane 6). In contrast, the serum of the MCTD patient exhibited strong reactivity with fragment 1-170 (lane 4 ), but no reactivity with fragments 91-182 (lane 5) and 105-182 (lane 6).

In Fig. 2 the combined results of the immunoblot analyses obtained with 12 different fragments of hnRNP-A2 are summarized. For all sera, reactivity of the two recombinant fulllength proteins was comparable to that of the natural antigen (and none of the sera were reactive with the MS-2 polymerase fusion part alone [not shown]). Comparable reactivity was seen with fragment 1-192 (lacking the glycine-rich auxiliary domain). With the exception of two SLE sera, all sera recognized fragment 1-182 although the reactivities of some sera (four RA and four SLE) appeared somewhat weaker $(++)$ than with fragment 1-192 $(+++)$. Further COOH-terminal truncations gradually reduced or abolished all reactivities: thus, fragment 1-178 (not shown in Fig. 2) terminating one amino acid downstream of RBD II was considerably less reactive with most sera $(+$ or ++$)$ than fragment 1-182, and fragment 1-170 was recognized by only three RA and four SLE sera, whereas six of the eight MCTD sera were still fully reactive. Eight of these sera (two RA, two SLE, four MCTD) recognized also a fragment of 147 amino acids but only a single SLE serum was
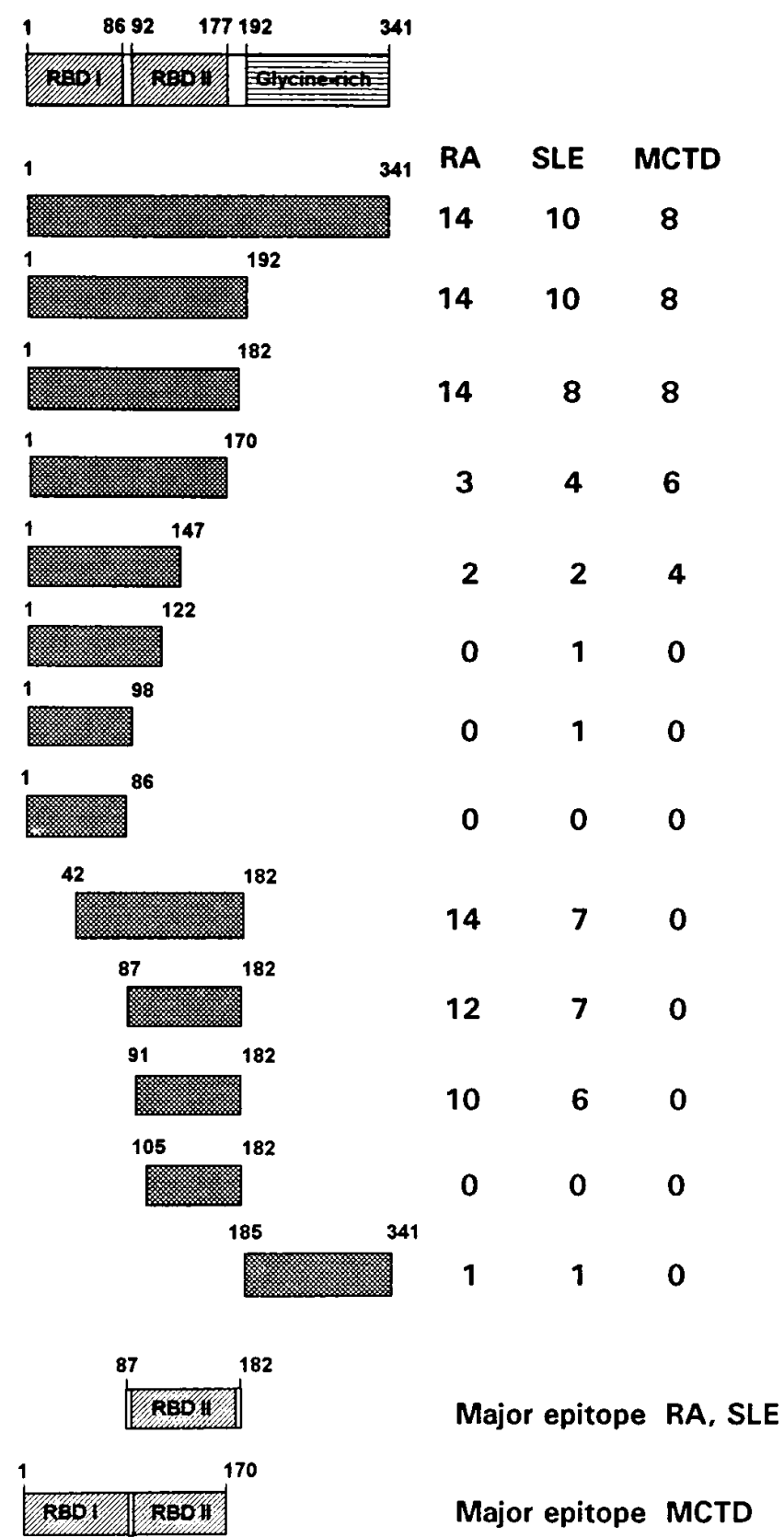

Figure 2. Summary of the immunoblotting results obtained with recombinant fragments of hnRNP-A2. The two RNA binding domains (RBD I, RBD II) and the glycine-rich auxiliary domain are schematically drawn; numbers above the hnRNP-A2 model designate the amino acid positions at the border of each domain. The amino acids bordering the deletion mutants are indicated. Numbers of reactive sera are shown in the table at the right. The major epitope recognized by most RA and SLE sera was located between amino acids 87 and 182, the major epitope targeted by MCTD sera comprised presumably the complete RBD I plus most of RBD II. Minor epitopes recognized by some RA and SLE patients were identified between amino acids 87-147 (two RA, one SLE), 42-182 (two RA), 87-170 (one SLE), 87-98 (one SLE), 1-170 (one SLE), and 185-341 (one RA, one SLE).

weakly reactive $(+)$ with a $122-$ amino acid fragment. Taken together, removal of the glycine-rich auxiliary domain had no significant effect on binding of autoantibodies whereas $\mathrm{COOH}$-terminal deletions into the RBD II region led to sub- 
stantial losses of reactivity with the majority of sera, particularly with those from RA and SLE patients. No serum was reactive with the first RBD (RBD I) alone, and only one RA and one SLE serum showed weak reactivity $(+)$ with the $\mathrm{COOH}-$ terminal part of hnRNP-A2 (185-341), suggesting that this domain contained only a minor epitope recognized by a very small proportion of sera.

Although these data clearly demonstrated the importance of COOH-terminal amino acids of RBD II for binding of autoantibodies the actual size of the epitopes could not be deduced from them. Therefore a series of $\mathrm{NH}_{2}$-terminal deletion mutants of fragment 1-182 was constructed. Removal of 41 amino acids from the $\mathrm{NH}_{2}$ terminus of RBD I did not, except for one SLE serum, affect binding of RA and SLE sera; in sharp contrast, all sera from MCTD patients became nonreactive. Fragment 87-182 containing RBD II plus five flanking amino acids at the $\mathrm{COOH}$ and $\mathrm{NH}_{2}$ termini, respectively, was still recognized by the majority of RA and SLE sera, most of which were also reactive with fragment 91-182. However, further $\mathrm{NH}_{2}$-terminal truncations of RBD II (fragment 105-182) completely abolished the reactivity of all sera. These results allowed to conclude $(a)$ that $\mathrm{NH}_{2}$-terminal sequences of RBD I were involved in binding of autoantibodies from patients with MCTD (but not from patients with RA or SLE), and (b) that $\mathrm{NH}_{2}$-terminal sequences of RBD II were essential for recognition by autoantibodies from patients with RA or SLE (but not from patients with MCTD).

In summary, these experiments characterized two major antigenic regions which were discontinuous and therefore presumably conformational: the first one contained the complete RBD II region and was recognized by most RA and SLE sera but not by MCTD sera; the other one comprised presumably the complete RBD I plus large parts of RBD II and was targeted predominantly by MCTD sera.

Binding of oligoribonucleotides to hnRNP-A2. As both major autoepitopes were located in a region of hnRNP-A2 assumed to be involved in binding of RNA, we were interested to investigate whether the autoantibodies would be able to affect interaction of hnRNP-A2 with RNA, and thus to interfere with the function of the antigen. In these experiments the oligoribonucleotide $\mathrm{r}(\mathrm{UUAGGG})_{4}$ was primarily used as RNA substrate since high-affinity binding of this sequence [or the corresponding DNA sequence (TTAGGG) $)_{4}$, respectively] to natural hnRNP-A2 had been demonstrated previously (24). The specificity of this interaction was shown to depend on the first four nucleotides (UUAG) since mutations of any of these bases led to considerable decreases in affinity (25).

In a first series of experiments the interaction between hnRNP-A2 and RNA was investigated by competition assays in which recombinant hnRNP-A2 was incubated with a mixture of ${ }^{32} \mathrm{P}$-labeled $\mathrm{r}(\mathrm{UUAGGG})_{4}$ and a 50 -fold excess of one of four unlabeled oligoribonucleotides: $(a) \mathrm{r}(\mathrm{UUAGGG})_{4}$ as a positive control; $(b)$ a sequence containing two r(UUAG) motives known to bind with high affinity to hnRNP-A1 ("A1 winner sequence") (26); (c) a sequence containing one r(UUAG) motif corresponding to the $3^{\prime}$ splice site of the first intron of the human $\beta$ globin gene; and $(d)$ a sequence derived from within this intron lacking such a motif. Binding of labeled r(UUAGGG $)_{4}$ was determined in two ways: $(a)$ by UV crosslinking assays in which, upon UV irradiation, bound oligonucleotides were covalently linked to the protein followed by SDS-PAGE analysis; and $(b)$ by gel retardation assays in
Table I. Competition of Oligoribonucleotides for Binding to $h n R N P-A 2$

\begin{tabular}{llc}
\hline \multicolumn{1}{c}{ Name } & \multicolumn{1}{c}{ Sequence } & \% Inhibition \\
\hline A2 winner* & (UUAGGG) $_{4}$ & 97 \\
A1 winner & UAUGUUAGGGACUUAGGGUG & 87 \\
3' splice site & CCACCCUUA $\downarrow$ GGCUGCUGGUG & 25 \\
Intron & GAUCACUUGUGUCAACACAG & 0 \\
& & \\
\hline
\end{tabular}

The binding of oligoribonucleotides to hnRNP-A2 was investigated in a competition binding assay using unlabeled oligoribonucleotides in a 50fold molar excess over ${ }^{32} \mathrm{P}$-labeled $\mathrm{r}(\mathrm{UUAGGG})_{4}$. Data are given as percent inhibition of ${ }^{32} \mathrm{P}-\mathrm{r}(\mathrm{UUAGGG})_{4}$ binding in a UV cross-linking assay. *Unlabeled $\mathrm{r}(\mathrm{UUAGGG})_{4}($ A2 winner $) ;$; sequence binding with high affinity to hnRNP-A1 (A1 winner) (26); ${ }^{\S}$ sequence corresponding to the $3^{\prime}$ splice site of the first intron of the $\beta$ globin gene, the arrow indicates the intron-exon boundary; "sequence derived from within the first intron of the $\beta$ globin gene. The UUAG motives are underlined.

which RNA-protein complexes were resolved on nondenaturing gels. Both assays yielded comparable data. As shown in Table I, unlabeled r(UUAGGG $)_{4}$ and also the A1 winner sequence were the most efficient competitors (i.e., binders). The $3^{\prime}$ splice site sequence of the $\beta$ globin intron showed moderate competition (i.e., binding capacity) whereas the intron sequence did not bind. These results allowed us to conclude that the presence of $\mathrm{r}(\mathrm{UUAG})$ motives was indeed required for sequence specific interactions between hnRNP-A2 and RNA as suggested previously by other investigators $(24,25)$.

Localization of the binding site for the oligoribonucleotide $r(U U A G G G)_{4}$. To characterize the binding site of $\mathrm{r}(\mathrm{UUAGGG})_{4}$ two different experimental approaches were used: ( $a$ ) UV cross-linking (Fig. $3 A$ ), and (b) Northwestern blot analysis where the binding assay was performed with fragments blotted to nitrocellulose (Fig. $3 \mathrm{~B}$ ). The latter assay required long renaturation times $(18-24 \mathrm{~h})$ to allow efficient binding of RNA to the immobilized proteins. (It should be noted that such long renaturation times were not required for binding of antibodies; nevertheless, their reactivity was considerably enhanced under these conditions.) Similar results were obtained in both experiments; no difference in binding was seen between the two full-size (nonfusion and fusion) proteins (Fig. 3, $A$ and $B$, lanes 1 and 2). Compared to these two proteins, the binding capacity of fragment $1-182$ (lane 3 ) was $\sim 80 \%$ and that of fragment 91-182 (lane 5) was $\sim 60 \%$. No binding was seen with fragments 1-170 (lane 4) and 105-182 (lane 6) nor with the COOH-terminal fragment 185-341 (not shown). Similar to RA and SLE autoantibodies, the oligoribonucleotide showed rather weak affinity for mutant $1-178$ (only 10\% binding capacity) although this fragment still contained both RBDs (not shown). Thus, the RNA binding pattern was very similar to the pattern observed with autoantibodies from patients with RA or SLE indicating that the binding sites for RNA and autoantibodies were similar.

Inhibition of RNA binding by anti-A2/RA33 antibodies. The data from the epitope mapping and RNA binding studies provided clear evidence that the complete RBD II of hnRNPA2 was essential and also sufficient for binding of both autoantibodies (from RA and SLE patients) and RNA. To further analyze the relationship between the two binding regions, the 


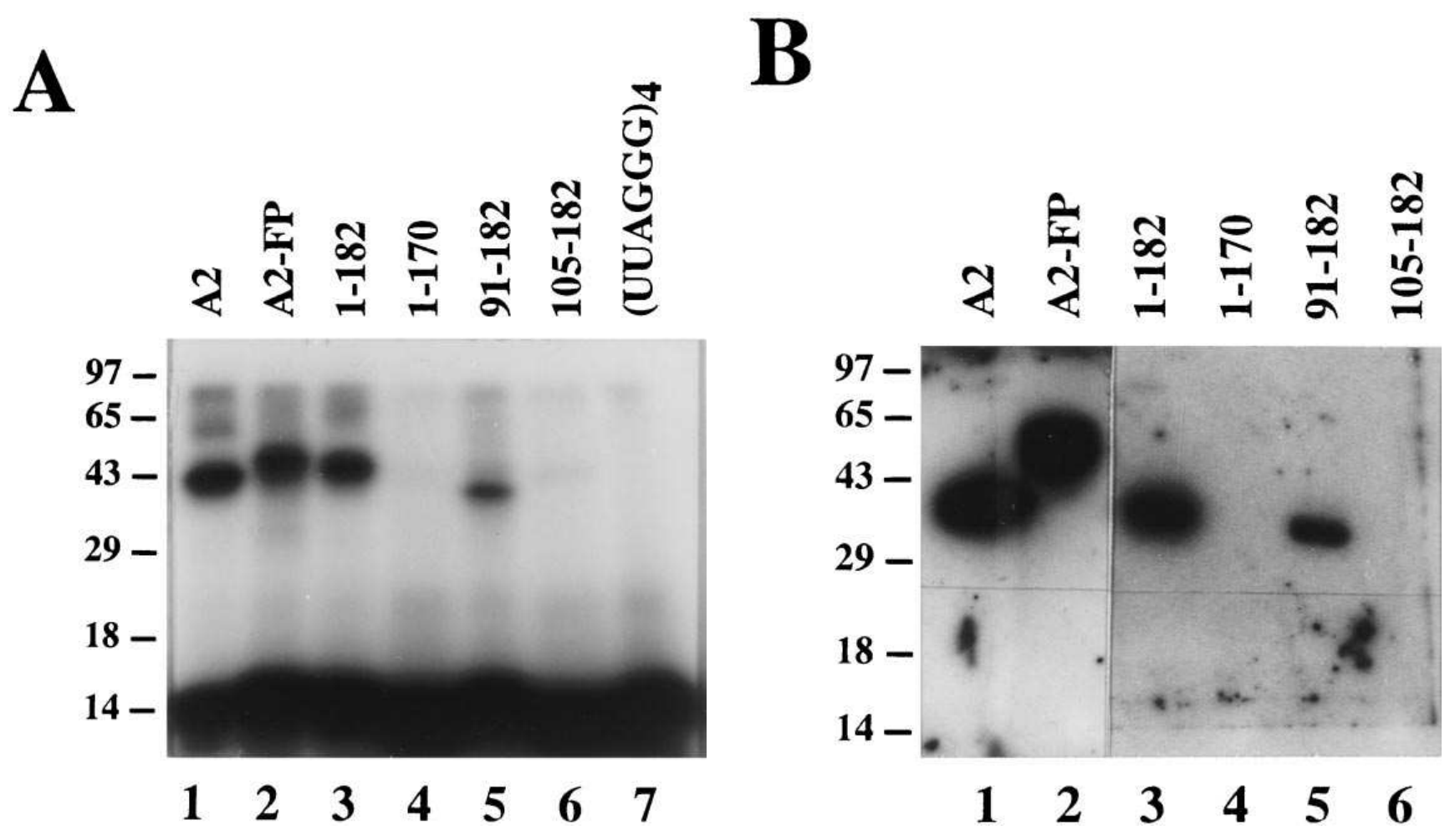

Figure 3. Binding of hnRNP-A2 and deletion mutants (same as in Fig. 1$)$ to the ${ }^{32}$ P-labeled oligoribonucleotide r(UUAGGG) $)_{4}$. $(A)$ UV crosslinking assay: cross-linked products were resolved by SDS-PAGE and autoradiographed. Lane 7 contained only the oligoribonucleotide. $(B)$ Northwestern assay: proteins were separated by SDS-PAGE, blotted onto nitrocellulose, incubated with the oligonucleotide, and finally autoradiographed.

potency of anti-A2/RA33 antibodies to inhibit RNA binding was investigated. Using antibodies affinity-purified from patients' sera in a UV cross-linking assay, a dose-dependent decrease in oligoribonucleotide binding to hnRNP-A2 was observed with half-maximal inhibition at an anti-A2/RA33 antibody concentration of $\sim 5 \mu \mathrm{g} / \mathrm{ml}$, whereas (affinity-purified) anti-Sm antibodies were ineffective (Fig. 4). Very similar results were obtained with antibodies from patients with RA and SLE, respectively. In contrast, anti-A2/RA33 antibodies from patients with MCTD did not inhibit binding of RNA (not shown). Comparable data were obtained in a gel retardation assay and in a Northwestern blotting experiment (not shown). These results strongly suggested that the binding sites for RNA and autoantibodies from patients with RA and SLE were overlapping or even identical. Furthermore, these data allowed us to estimate that the affinity of the autoantibody was

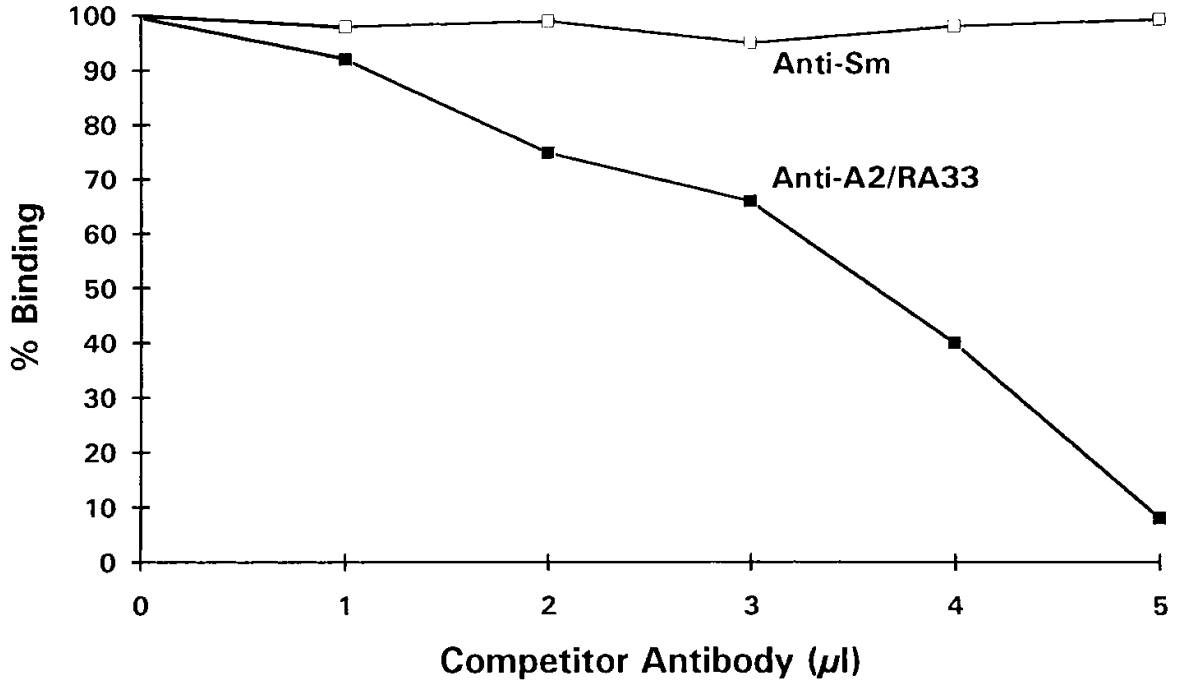

Figure 4. Competition between anti-A2/ RA33 autoantibodies and r(UUAGGG) $)_{4}$ for binding to hnRNP-A2. Increasing amounts of anti-A2/RA33 autoantibodies affinity-purified from sera of patients with RA or SLE were added to constant amounts of labeled r(UUAGGG) $)_{4}$ and recombinant nonfusion hnRNP-A2. An affinity-purified anti-Sm antibody from an anti-A2/RA33 positive SLE patient was used as control. After UV cross-linking the complex was separated by SDS-PAGE and RNA binding was quantitated by electronic autoradiography. A typical result obtained with affinity-purified anti-A2/ RA33 antibodies from a patient with RA is shown; antibodies derived from a patient with SLE gave a very similar result; no inhibition was seen by anti-Sm antibodies. 
at least one order of magnitude higher than the affinity determined for the interaction of hnRNP-A2 with $\mathrm{r}(\mathrm{UUAGGG})_{4}$ $\left(K_{\mathrm{d}}=2 \times 10^{-6} \mathrm{M}\right)$.

\section{Discussion}

The nuclear protein hnRNP-A2 (the RA33 antigen) belongs to the group of spliceosome-associated autoantigens (23). However, in contrast to the spliceosomal snRNPs, it is targeted not only by patients with SLE and MCTD, who characteristically generate an autoimmune response to the spliceosome, but also by patients with RA $(13,14,27)$. The detailed epitope mapping study performed with sera from patients with RA, SLE, and MCTD reveals that the major autoepitopes of hnRNP-A2 are discontinuous. Thus, a sequence of $\sim 95$ amino acids containing the complete RBD II was required for binding of the majority of anti-A2/RA33 autoantibodies from patients with RA and SLE. This indicates that the epitope(s) targeted by these antibodies is conformation dependent but it does not necessarily mean that antibodies from RA and SLE patients bind to the same site within RBD II. Interestingly, this epitopic region was not recognized by autoantibodies from patients with MCTD which bound to a much larger epitope comprising RBD I and at least two-thirds of RBD II. This finding was somewhat surprising since, given that MCTD and SLE are clinically and serologically more closely related to each other than to RA, one would rather have expected SLE and MCTD patients to recognize similar epitopes.

Serologically, MCTD is characterized by high-titer antibodies to U1-snRNP (without concomitant anti-Sm), particularly to the U1-70K protein. This serologic picture is not specific for MCTD but can also be seen in some patients with SLE $(1,2)$. However, the one SLE patient in this study showing MCTD-like anti-U1-snRNP reactivity did not recognize the major MCTD epitope. Thus, the specific epitope recognition by anti-A2/RA33 autoantibodies seems to be a novel serological feature of MCTD which distinguishes it from SLE. This would be in line with the reported preferential epitope recognition of the U1-snRNP associated U1-A antigen by MCTD sera (28). In light of the still ongoing debate of whether MCTD represents a disease entity or rather an overlap of several connective tissue diseases $(21,29-32)$, these findings support the notion that MCTD may be considered a distinct connective tissue disease.

Remarkably, apart from two exceptions, binding of autoantibodies to the COOH-terminal half of hnRNP-A2 (i.e., the auxiliary domain) was not observed. This part of the antigen contains almost $50 \%$ glycine residues and has some significant homologies with other glycine-rich proteins, such as collagen, keratin, or EBV nuclear antigen-1 (33). Since sera from RA patients frequently contain autoantibodies to these proteins, cross-reactivity with epitopes in the auxiliary domain as described for autoantibodies to hnRNP-A1 could have been possible (34). However, in our initial study we found no evidence for an association of anti-A2/RA33 with antibodies to EBV nuclear antigen-1 (5). Thus, cross-reactivity with other glycine-rich antigens is unlikely to play a role in the development of the anti-A2/RA33 autoimmune response. To explain the lack of reactivity of the auxiliary domain, one should take into account that this region has been shown to closely interact with other proteins (35) and therefore may be largely inaccessible (hidden) for (auto)immune recognition.
To investigate the relationship between the recognition site of autoantibodies and the biological function of the antigen, RNA binding studies were performed which confirmed that RNA sequences containing the motif $r(U U A G)$ were preferentially bound by hnRNP-A2 $(24,25)$. The highest affinity was observed with an oligonucleotide containing four repeats of this motif. A sequence corresponding to the $3^{\prime}$ splice site of the first intron of the $\beta$ globin pre-mRNA which contained only one $\mathrm{r}(\mathrm{UUAG})$ motif, still showed significant binding affinity [although approximately one order of magnitude less than $\left.\mathrm{r}(\mathrm{UUAGGG})_{4}\right]$, whereas a sequence derived from within the $\beta$ globin intron lacking this motif did not bind. Similar results have been reported for the closely related hnRNP-A1 indicating that the intron-exon acceptor sites may be biologically relevant binding structures for both proteins (26). This may be important for their suggested role in regulating alternative splicing of (certain) pre-mRNAs (17).

Mapping the binding region for these oligonucleotides clearly showed that RBD II alone was essential and sufficient for binding and that $\mathrm{NH}_{2}$ - or $\mathrm{COOH}$-terminal deletions of RBD II completely abolished RNA binding activity. Thus, the binding behavior of RNA was remarkably similar to that of autoantibodies from patients with RA or SLE. As with autoantibodies, neither RBD I alone nor the $\mathrm{COOH}$-terminal part of the protein showed affinity for the oligonucleotides tested. However, binding efficiency was lower when these parts of the protein were deleted, suggesting an indirect participation in RNA binding.

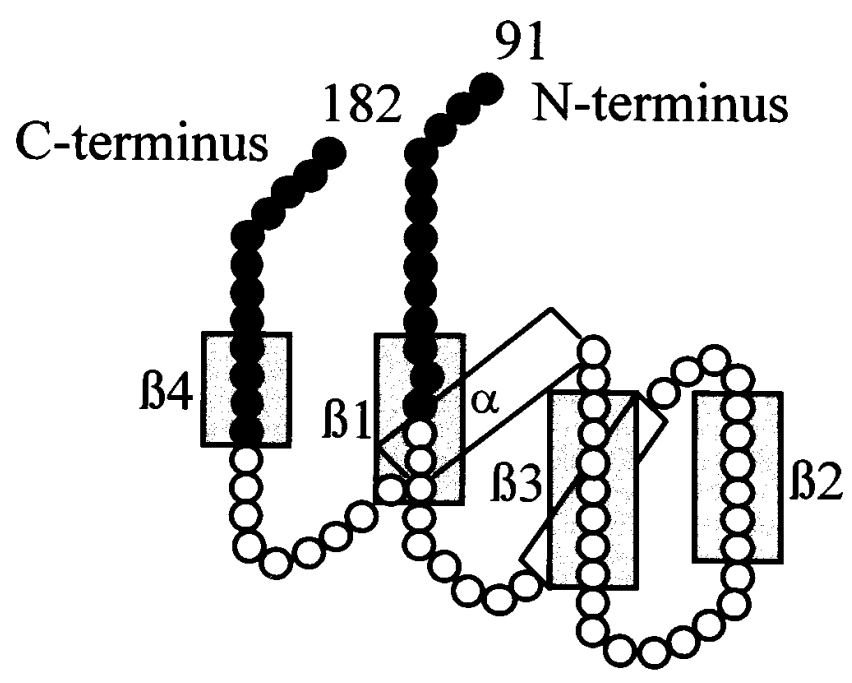

Figure 5. Structural model of the second RNA binding domain (RBD II) of hnRNP-A2 (37-39). In this model, a four-stranded antiparallel $\beta$ sheet (gray boxes) is packed against two perpendicularly oriented $\alpha$ helices (white boxes). The two most highly conserved sequences, the octameric RNP1 and the hexameric RNP2, are juxtaposed on the central $\beta 1$ and $\beta 3$ strands making direct contact with RNA which is not dependent on the RNA sequence. RNA binding specificity resides in the most variable regions, particularly in the loops and the $\mathrm{NH}_{2}$ and $\mathrm{COOH}$ termini. Black circles represent amino acids, the presence of which was essential for binding of autoantibodies from RA and SLE patients as well as for the interaction with the oligoribonucleotide r(UUAGGG) $)_{4}$. Thus, neither autoantibodies nor RNA bound to a fragment lacking the $\mathrm{NH}_{2}$-terminal amino acids, and only few sera bound to a fragment where the $\mathrm{COOH}$-terminal amino acids were truncated. 
Thus, deletions of the $\mathrm{NH}_{2}$ - or $\mathrm{COOH}$-terminal portions of RBD II had comparable consequences both for antibody recognition and for RNA binding, demonstrating that these sequences were involved in both binding activities and that intact conformation was required for binding. Remarkably, in a generalized structural model developed for RBDs of the type present in hnRNP-A2, the $\mathrm{NH}_{2}$ - and $\mathrm{COOH}$-terminal regions are in close proximity to each other $(36,37)$ (Fig. 5). This, together with the findings reported here, is suggestive of a direct interaction of these sequences both with antibodies and RNA. Regarding the interaction with RNA, it has been shown that general RNA binding activity is dependent on the most conserved sequences located in a compact structure composed of four antiparallel $\beta$ sheets whereas specificity is conferred by the less conserved flanking regions as well as by loop sequences linking the $\beta$ sheets (37-39). Of interest, the loop structures of the $\mathrm{NH}_{2}$-terminal RBD of the U1-A antigen have been shown recently to contain important autoantibody binding sites (40) underlining once more the importance of the nonconserved sequences for binding specificity. Finally, the competition between anti-A2/RA33 autoantibodies and RNA for binding to hnRNP-A 2 can be considered another strong indication that the antibodies recognize the same or a very similar structure as the natural ligand RNA. Thus, at least in vitro these antibodies could potentially interfere with the biological activity of the target protein, a phenomenon commonly observed with disease-related autoantibodies (41). Remarkably, competition was only seen with antibodies recognizing the complete RBD II, i.e., those derived from patients with RA and SLE, and not with those from patients with MCTD directed to RBD I and the $\mathrm{NH}_{2}$-terminal portion of RBD II.

The differential epitope and antigen recognition patterns observed for RA (A2-RBD II only), SLE (A2-RBD II, Sm, and U1-snRNP), and MCTD (A2-RBD I+II, U1-snRNP) underscores, once more, the view of a highly specific antigendriven autoimmune response. It is a remarkable and not yet understood phenomenon that in RA this immune response is directed only to the two hnRNP-A proteins (and their alternatively spliced variants, the hnRNP-B proteins) but not to other components of the spliceosome as in SLE and MCTD. The results of the epitope mapping study cannot provide an explanation since similar structures were obviously targeted by patients with RA and SLE. However, the epitopes may not be accessible when the antigen is associated with other proteins in the spliceosome. This is indicated by the inability of patients' antibodies to precipitate hnRNP-A2 from nuclear extracts, in contrast to monoclonal antibodies (15, our unpublished observation). Thus, due to such inaccessibility, intermolecular spreading of the autoimmune response to other components of the spliceosome, as has been seen in animals immunized with snRNP components or even small peptides, could be impaired in RA, in contrast to SLE (42-44). In this context it may be important to note that hnRNP-A proteins can dissociate from the spliceosome and shuttle between the nucleus and the cytosol (16). Therefore, non-spliceosome-associated hnRNP-A2 might be the actual target for autoantibodies in RA which, however, would be unable to induce loss of tolerance to other spliceosomal components by virtue of their inability to recognize the antigen when complexed with the spliceosome. In contrast, in SLE and MCTD, where the anti-hnRNP and anti-snRNP reactivities appear to be closely linked (13), the anti-hnRNP response might be secondary to the anti-snRNP response. This hypothesis can be tested by immunizing animals with either hnRNP or snRNP proteins and preliminary data obtained with lupus-prone MRL/lpr mice indicate that in this model autoantibodies to snRNP antigens may indeed precede antibodies to hnRNP antigens (45, our unpublished observation).

In conclusion, the RNA binding domains of hnRNP-A2 have been identified as the main antigenic regions targeted by anti-A2/RA33 autoantibodies. Structurally similar RNA binding regions have been shown to contain major epitopes also in other well-defined autoantigens such as U1-70K, U1-A, or La (46). It is still poorly understood why so many autoantigens in rheumatic diseases are components of RNP complexes $(3,47)$. Assuming an antigen-driven autoimmune process, it is conceivable that RNA binding structures are more exposed and therefore more immunogenic than other parts of the protein.

\section{Acknowledgments}

We thank Chris Burd and Gideon Dreyfuss for their kind gift of the hnRNP-A2 cDNA; Christoph Zimmermann for providing clinical data; and Walther van Venrooij for his critical comments and encouraging discussions.

This work was supported by grant P-9152 MED from the Austrian "Fonds zur Förderung der Wissenschaftlichen Forschung."

\section{References}

1. von Mühlen, C.A., and E.M. Tan. 1995. Autoantibodies in the diagnosis of systemic rheumatic diseases. Semin. Arthritis Rheum. 24:323-358.

2. Peng, S.L., J.A. Hardin, and J. Craft. 1997. Antinuclear antibodies. In Textbook of Rheumatology. W.N. Kelley, E.D. Harris, S. Ruddy, and C.B. Sledge, editors. W.B. Saunders Company, Philadelphia, PA. 250-266.

3. van Venrooij, W., and G.J.M. Pruijn. 1995. Ribonucleoprotein complexes as autoantigens. Curr. Opin. Immunol. 7:819-824.

4. Tighe, H., and D.A. Carson. 1997. Rheumatoid factors. In Textbook of Rheumatology. W.N. Kelley, E.D. Harris, S. Ruddy and C.B. Sledge, editors. W.B. Saunders Company, Philadelphia, PA. 241-249.

5. Hassfeld, W., G. Steiner, K. Hartmuth, G. Kolarz, O. Scherak, W. Graninger, N. Thumb, and J.S. Smolen. 1989. Demonstration of a new antinuclear antibody (anti-RA33) that is highly specific for rheumatoid arthritis. Arthritis Rheum. 32:1515-1520.

6. Hassfeld, W., G. Steiner, W. Graninger, G. Witzmann, H. Schweitzer, and J.S. Smolen. 1993. Autoantibody to the nuclear antigen RA33: a marker for early rheumatoid arthritis. Br. J. Rheumatol. 32:199-203.

7. Youinou, P., and G. Serre. 1995. The antiperinuclear factor and antikeratin antibody systems. Int. Arch. Allergy Immunol. 107:508-518.

8. Sebbag, M., M. Simon, C. Vincent, C. Masson-Bessière, E. Girbal, J.J. Durieux, and G. Serre. 1995. The antiperinuclear factor and the so-called antikeratin antibodies are the same rheumatoid arthritis-specific autoantibodies. $J$. Clin. Invest. 95:2672-2679.

9. Dèspres, N., G. Boire, F.J. Lopez-Longo, and H.A. Menard. 1994. The Sa system: a novel antigen-antibody system specific for rheumatoid arthritis. $J$. Rheumatol. 21:1027-1033.

10. Steiner, G., K. Hartmuth, K. Skriner, I. Maurer-Fogy, A. Sinski, E. Thalmann, W. Hassfeld, A. Barta, and J.S. Smolen. 1992. Purification and partial sequencing of the nuclear autoantigen RA33 shows that it is indistinguishable from the A2 protein of the heterogeneous nuclear ribonucleoprotein complex. J. Clin. Invest. 90:1061-1066.

11. Dreyfuss, G., M.J. Matunis, S. Pinol-Roma, and C.G. Burd. 1993. hnRNP proteins and the biogenesis of mRNA. Annu. Rev. Biochem. 62:289-321.

12. Astaldi-Ricotti, G.C.B., M. Bestagno, A. Cerini, R. Negri, R. Caporali, F. Cobianchi, M. Longhi, and C. Montecucco. 1989. Antibodies to hnRNP core protein A1 in connective tissue diseases. J. Cell. Biochem. 40:1-5.

13. Hassfeld, W., G. Steiner, A. Studnicka-Benke, K. Skriner, W. Graninger, I. Fischer, and J.S. Smolen. 1995. Autoimmune response to the spliceosome. An immunologic link between rheumatoid arthritis, mixed connective tissue disease, and systemic lupus erythematosus. Arthritis Rheum. 38:777-785.

14. Meyer, O., F. Tauxe, D. Fabregas, C. Gabay, M. Goycochea, T. Haim, A. Elias, and M.F. Kahn. 1993. Anti-RA33 antinuclear autoantibody in rheumatoid arthritis and mixed connective tissue disease: comparison with antikeratin and antiperinuclear antibodies. Clin. Exp. Rheumatol. 11:473-478.

15. Piñol-Roma, S., Y.D. Choi, M.J. Matunis, and G. Dreyfuss. 1988. Immunopurification of heterogeneous nuclear ribonucleoprotein particles reveals an assortment of RNA-binding proteins. Genes Dev. 2:215-227. 
16. Piñol-Roma, S., and G. Dreyfuss. 1992. Shuttling of pre-mRNA binding proteins between nucleus and cytoplasm. Nature (Lond.). 355:730-732.

17. Mayeda, A., S.H. Munroe, J.F. Caceres, and A.R. Krainer. 1994. Function of conserved domains of hnRNP A1 and other hnRNP A/B proteins. EMBO (Eur. Mol. Biol. Organ.) J. 13:5483-5495.

18. Burd, C.G., M.S. Swanson, M. Görlach, and G. Dreyfuss. 1989. Primary structures of the heterogeneous nuclear ribonucleoprotein A2, B1, and C2 proteins: a diversity of RNA binding proteins is generated by small peptide inserts. Proc. Natl. Acad. Sci. USA. 86:9788-9792.

19. Arnett, F.C., S.M. Edworthy, D.A. Bloch, D.J. McShane, J.F. Fries, N.S. Cooper, L.A. Healey, S.R. Kaplan, M.H. Liang, H.S. Luthra, et al., 1988. The American Rheumatism Association 1987 revised criteria for the classification of rheumatoid arthritis. Arthritis Rheum. 31:315-324.

20. Tan, E.M., A.S. Cohen, J.F. Fries, A.T. Masi, D.J. McShane, N.F. Rothfield, J.G. Schaller, N. Talal, and R.J. Winchester. 1982. The 1982 revised criteria for the classification of systemic lupus erythematosus. Arthritis Rheum. 25: 1271-1277.

21. Alarcón-Segovia, D., and M. Villareal 1987. Classification and diagnostic criteria for mixed connective tissue diseases. In Mixed Connective Tissue Diseases and Anti-nuclear Antibodies. R. Kasukawa and G.C. Sharp, editors. Elsevier, Amsterdam. 33-40.

22. Strebel, K., E. Beck, K. Strohmaier, and H. Schaller. 1986. Characterization of foot-and mouth disease gene products with antisera against bacterially synthesised fusion proteins. J. Virol. 57:983-991.

23. Steiner, G. 1994. The A2 protein of the heterogeneous nuclear ribonucleoprotein (hnRNP-A2)/RA33. In Manual of Biological Markers of Disease. Vol. B. W.J. van Venrooij and R.N. Maini, editors. Kluwer Academic Publishers, Dordrecht/Boston/London. 1.3/1-9.

24. McKay, S.J., and H. Cooke. 1992. hnRNP A2/B1 binds specifically to

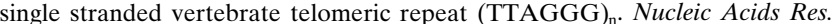
20:6461-6464.

25. Ishikawa, F., M.J. Matunis, G. Dreyfuss, and T.R. Cech. 1993. Nuclear proteins that bind the pre-mRNA $3^{\prime}$ splice site sequence r(UUAG/G) and the human telomeric DNA sequence d(TTAGGG)n. Mol. Cell. Biol. 13:4301-4310.

26. Burd, C.G., and G. Dreyfuss. 1994. RNA binding specificity of hnRNP A1: significance of hnRNP A1 high-affinity binding sites in pre-mRNA splicing. EMBO (Eur. Mol. Biol. Organ.) J. 13:1197-1204.

27. Aho, K., G. Steiner, P. Kurki, L. Paimela, M. Leirisalo Repo, T. Palosuo, and J.S. Smolen. 1993. Anti-RA33 as a marker antibody of rheumatoid arthritis in a Finnish population. Clin. Exp. Rheumatol. 11:645-647.

28. Barakat, S., J.P. Briand, N. Abuaf, M.H. van Regenmortel, and S. Muller. 1991. Mapping of epitopes on U1 snRNP polypeptide A with synthetic peptides and autoimmune sera. Clin. Exp. Immunol. 86:71-78.

29. Sharp, G.C, W.S. Irvin, E.M. Tan, G.R. Gould, and H.R. Holman. 1972. Mixed connective tissue disease: an apparently distinct rheumatic disease syndrome associated with a specific antibody to an extractable nuclear antigen. Am. J. Med. 52:148-159.

30. McHugh, N., I. James, and P. Maddison. 1990. Clinical significance of antibodies to a $68 \mathrm{kDa}$ U1RNP polypeptide in connective tissue disease. $J$. Rheumatol. 17:1320-1328.

31. Kallenberg, C.G. 1995. Overlapping syndromes, undifferentiated connective tissue disease, and other fibrosing conditions. Curr. Opin. Rheumatol. 7:
$568-573$.

32. Citera, G., M.A. Lazaro, and J.A. Maldonado Cocco. 1995. Mixed connective tissue disease: fact or fiction? Lupus. 4:255-257.

33. Steiner, G., K. Skriner, and J.S. Smolen. 1996. Autoantibodies to the A/B proteins of the heterogeneous nuclear ribonucleoprotein (hnRNP) complex-novel tools for the diagnosis of rheumatic diseases. Int. Arch. Allergy Immunol. 111:314-319.

34. Montecucco, C., R. Caporali, C. Negri, F. de Gennaro, A. Cerino, M. Bestagno, F. Cobianchi, and G.C. Astaldi-Ricotti. 1990. Antibodies from patients with rheumatoid arthritis and systemic lupus erythematosus recognize different epitopes of a single heterogeneous nuclear RNP core protein. Possible role of cross-reacting antikeratin antibodies. Arthritis Rheum. 33:180-186.

35. Cartegni, L., M. Maconi, E. Morandi, F. Cobianchi, S. Riva, and G. Biamonti. 1996. hnRNP A1 selectively interacts through its gly-rich domain with different RNA-binding proteins. J. Mol. Biol. 259:337-348.

36. Garrett, D.S., P.J. Lodi, Y. Shamoo, K.R. Williams, G.M. Clore, and A.M. Gronenborn. 1994. Determination of the secondary structure and folding topology of an RNA binding domain of mammalian hnRNP A1 protein using three-dimensional heteronuclear magnetic resonance spectroscopy. Biochemistry. 33:2852-2858

37. Burd, C.G., and G. Dreyfuss. 1994. Conserved structures and diversity of functions of RNA-binding proteins. Science (Wash. DC). 265:615-621.

38. Görlach, M., M. Wittekind, R.A. Beckman, L. Mueller, and G. Dreyfuss. 1992. Interaction of the RNA-binding domain of the hnRNP C proteins with RNA. EMBO (Eur. Mol. Biol. Organ.) J. 11:3289-3295.

39. Birney, E., S. Kumar, and A.R. Krainer. 1993. Analysis of the RNA-recognition motif and RS and RGG domains: conservation in metazoan premRNA splicing factors. Nucleic Acids Res. 21:5803-5816.

40. James, J.A., and J.B. Harley. 1996. Human lupus anti-spliceosome A protein autoantibodies bind contiguous surface structures and segregate into two sequential epitope binding patterns. J. Immunol. 156:4018-4026.

41. Tan, E.M., Y. Muro, and K.M. Pollard. 1994. Autoantibody-defined epitopes on nuclear antigens are conserved, conformation-dependent and active site regions. Clin. Exp. Rheumatol. 12(Suppl. 11):27-31.

42. Fatenejad, S., M.J. Mamula, and J. Craft. 1993. Role of intermolecular/ intrastructural B and T-cell determinants in the diversification of autoantibodies to ribonucleoprotein particles. Proc. Natl. Acad. Sci. USA. 90:12010-12014.

43. Mamula, M.J., S. Fatenejad, and J. Craft. 1994. B cells process and present lupus autoantigens that initiate autoimmune T cell responses. J. Immunol. 152:1453-1461.

44. James, J.A., T. Gross, R.H. Scofield, and J.B. Harley. 1995. Immunoglobulin epitope spreading and autoimmune disease after peptide immunization: Sm B/B' -derived PPPGMRPP and PPPGIRGP induce spliceosome autoimmunity. J. Exp. Med. 181:453-461.

45. Steiner, G., J.S. Smolen, and H.M. Smith. 1995. Autoantibodies to hnRNP-A2 (RA33) in sera of MRL/lpr mice. Arthritis Rheum. 39:(Suppl.): S297. (Abstr.)

46. van Venrooij, W.J., and C.W. van Gelder. 1994. B cell epitopes on nuclear autoantigens. What can they tell us? Arthritis Rheum. 37:608-616.

47. Tan, E.M. 1991. Autoantibodies in pathology and cell biology. Cell. 67 : 841-842. 